\title{
Energy dispersive detector for white beam synchrotron x-ray fluorescence imaging
}

Matthew D. Wilson' , Thomas Connolley, Igor P. Dolbnya, Patrick S. Grant, Enzo Liotti, Andrew Lui, Andrew Malandain, Kawal Sawhney, Paul Seller, and Matthew C. Veale

Citation: AIP Conference Proceedings 1741, 050008 (2016); doi: 10.1063/1.4952928

View online: http://dx.doi.org/10.1063/1.4952928

View Table of Contents: http://aip.scitation.org/toc/apc/1741/1

Published by the American Institute of Physics 


\title{
Energy Dispersive Detector for White Beam Synchrotron $\mathrm{X}$-ray Fluorescence Imaging
}

\author{
Matthew D. Wilson ${ }^{1, \text { a) }}$, Thomas Connolley ${ }^{2}$, Igor P. Dolbnya ${ }^{3}$, Patrick S. Grant ${ }^{4}$, \\ Enzo Liotti ${ }^{4}$, Andrew Lui ${ }^{4}$, Andrew Malandain ${ }^{3}$, Kawal Sawhney ${ }^{3}$, Paul Seller ${ }^{1}$ and \\ Matthew C. Veale ${ }^{1}$ \\ ${ }^{1}$ Science and Technology Facilities Council, Rutherford Appleton Laboratory, Harwell Campus, UK. \\ ${ }^{2}$ Diamond Light Source, I12 Beamline, Harwell Campus, Didcot, Oxfordshire, UK. \\ ${ }^{3}$ Diamond Light Source, B16 Beamline, Harwell Campus, Didcot, Oxfordshire, UK \\ ${ }^{4}$ Department of Materials, University of Oxford Parks Road, Oxford, UK. \\ ${ }^{a)}$ Corresponding author: Matt.Wilson@stfc.ac.uk
}

\begin{abstract}
A novel, "single-shot" fluorescence imaging technique has been demonstrated on the B16 beamline at the Diamond Light Source synchrotron using the HEXITEC energy dispersive imaging detector. A custom made furnace with $200 \mu \mathrm{m}$ thick metal alloy samples was positioned in a white X-ray beam with a hole made in the furnace walls to allow the transmitted beam to be imaged with a conventional X-ray imaging camera consisting of a $500 \mu \mathrm{m}$ thick single crystal LYSO scintillator, mirror and lens coupled to an AVT Manta G125B CCD sensor. The samples were positioned $45^{\circ}$ to the incident beam to enable simultaneous transmission and fluorescence imaging. The HEXITEC detector was positioned at $90^{\circ}$ to the sample with a $50 \mu \mathrm{m}$ pinhole $13 \mathrm{~cm}$ from the sample and the detector positioned $2.3 \mathrm{~m}$ from pinhole. The geometric magnification provided a field of view of $1.1 \times 1.1 \mathrm{~mm}^{2}$ with one of the $80 \mathrm{x} 80$ pixels imaging an area equivalent to $13 \mu \mathrm{m}^{2}$. Al-Cu alloys doped with $\mathrm{Zr}$, Ag and Mo were imaged in transmission and fluorescence mode. The fluorescence images showed that the dopant metals could be simultaneously imaged with sufficient counts on all $80 x 80$ pixels within 60 s, with the X-ray flux limiting the fluorescence imaging rate. This technique demonstrated that it is possible to simultaneously image and identify multiple elements on a spatial resolution scale $\sim 10 \mu \mathrm{m}$ or higher without the time consuming need to scan monochromatic energies or raster scan a focused beam of X-rays. Moving to high flux beamlines and using an array of detectors could improve the imaging speed of the technique with element specific imaging estimated to be on a $1 \mathrm{~s}$ timescale.
\end{abstract}

\section{INTRODUCTION}

The ability to simultaneously image the distribution of elements throughout a sample, in-situ during an experiment with timing resolution is desirable in a number of fields, with metal alloy solidification used as an example in this case. X-ray transmission imaging techniques using metal alloy analogues or simple binary alloy models have been insightful studies but do not provide vital information on the elemental distribution in the liquid phase and cannot be used to simultaneously image many elements in the sample [1]. Conventional Synchrotron Xray Fluorescence (SXRF) can provide detailed maps of the elemental composition by raster scanning a focused Xray beam over a sample and measuring the fluorescence spectrum on a Ge or Si based detector with only a single or relatively small number of channels [2]. This can provide spatial resolution as low as $1 \mu \mathrm{m}$ but the scanning process prohibits time resolved experiments. Here, we use the HEXITEC energy resolving imaging detector [3] and a pinhole projection to collect X-ray fluorescence images of a metal alloy sample that is flood illuminated with a white X-ray beam. 


\section{EXPERIMENTAL ARRANGEMENT}

The experiments were conducted on the B16 Optics and Metrology Beamline at the Diamond Light Source, UK. A white beam from the bending magnet source with an area of $5 \times 5 \mathrm{~mm}^{2}$ was used as the primary beam to illuminate a metal alloy sample. The $200 \mu \mathrm{m}$ thick, 10x20mm alloy was made from an $\mathrm{Al}-\mathrm{Cu}$ matrix with $\mathrm{Ag}, \mathrm{Zr}$ and Mo added to the sample. The alloy was held in a custom made furnace at $45^{\circ}$ from the primary beam which heated the alloy in an Ar atmosphere. Conventional X-ray transmission radiographs were measured with a $500 \mu \mathrm{m}$ LYSO scintillator optically coupled to an AVG Manta G125B CCD camera via a $90^{\circ}$ mirror $17.5 \mathrm{~cm}$ behind the sample. The transmission camera provided at field of view (FoV) of $5 \mathrm{~mm} \times 3.8 \mathrm{~mm}$ and each pixel imaged an area equivalent to $3.96 \mu \mathrm{m} \times 3.96 \mu \mathrm{m}$. The X-ray fluorescence image was measured with the HEXITEC detector positioned $90^{\circ}$ to the primary beam. The fluorescence X-rays were projected through a $1 \mathrm{~mm} \mathrm{~W}$ plate with a $50 \mu \mathrm{m}$ pinhole positioned $13 \mathrm{~cm}$ from the sample and the HEXITEC detector was positioned $231 \mathrm{~cm}$ from the pinhole. The $80 \times 80$ pixels on a $250 \mu \mathrm{m}$ pitch of the HEXITEC detector provided a FoV of $1.1 \mathrm{~mm} \times 1.1 \mathrm{~mm}$ and each pixel measured an area of $13.75 \mu \mathrm{m}$. The detector had a $1 \mathrm{~mm}$ thick CdTe detector that was held at $20^{\circ} \mathrm{C}$ and biased at $-500 \mathrm{~V}$ and has previously been characterized with an average pixel energy resolution of $800 \mathrm{eV} \mathrm{FWHM}$ at $60 \mathrm{keV}$ [3]. The detector operates at a frame rate of $9 \mathrm{kHz}$ with a maximum detection rate of $10^{5}$ photons/s whilst still being able to correct for charge sharing [4]. A He filled flight tube with a $50 \mu \mathrm{m}$ thick Kapton windows was placed between the pinhole and the HEXITEC detector to reduce the attenuation of the fluorescence X-rays. A schematic of the experimental arrangement is shown in Figure 1, not to scale.

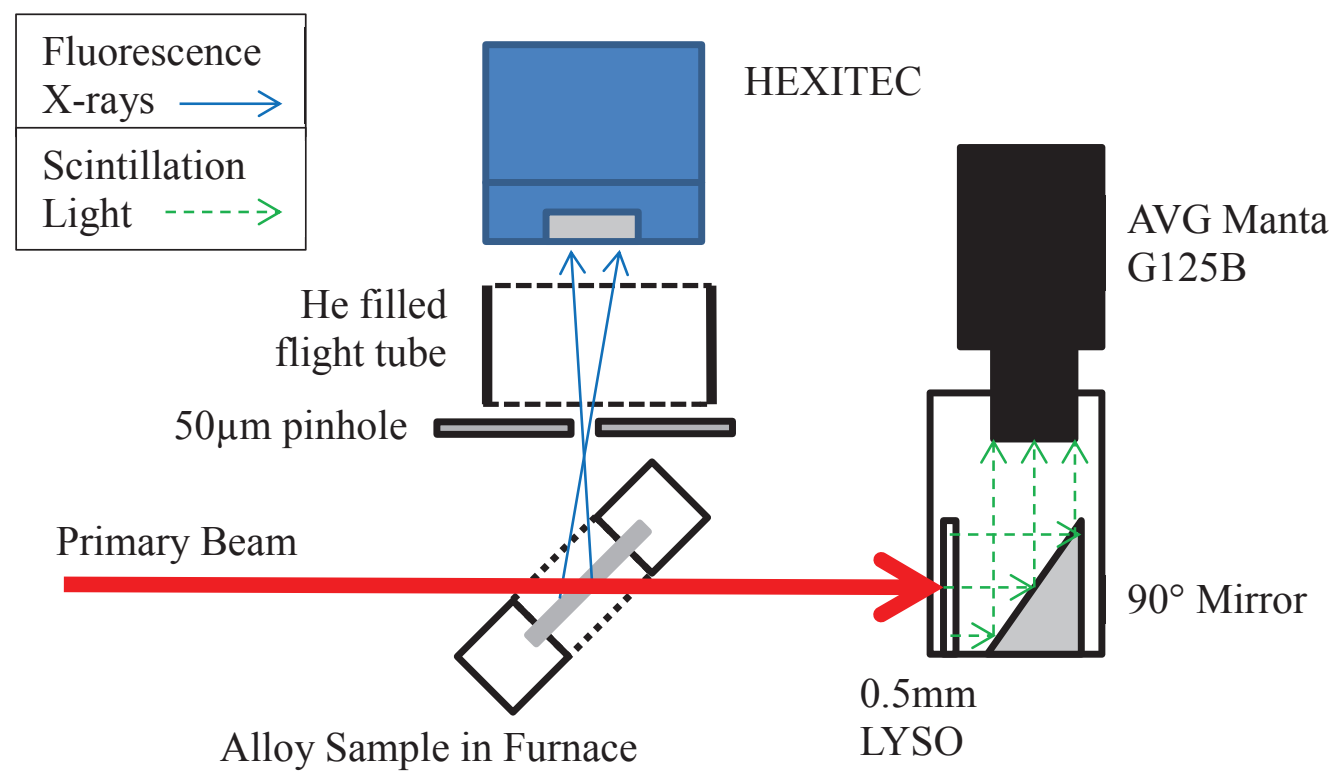

FIGURE 1. Schematic of the experimental arrangement, not to scale.

\section{RESULTS}

The experimental configuration was tested and aligned using a gold TEM grid (Gilder G200HS) that was $7.5 \mu \mathrm{m}$ thick and had $125 \mu \mathrm{m}$ pitch mesh, $12 \mu \mathrm{m}$ bar width and $113 \mu \mathrm{m}$ gaps. Figure 2 (a) shows the spectrum from $4 \times 4$ pixels in the region of the frame of the mesh (high intensity in Figure 2 (b)) were summed together to provide a spectrum with higher statistics. The Au L lines were visible between $9 \mathrm{keV}$ and $13 \mathrm{keV}$. The characteristic $\mathrm{Cd}$ and Te $\mathrm{K}$ lines from $23 \mathrm{keV}$ to $31 \mathrm{keV}$ due to the self-fluorescence of the detector and fluorescence from the $\mathrm{Lu}$ at $53 \mathrm{keV}$ to $62 \mathrm{keV}$ in the LYSO scintillator were observed. As the TEM grid is increasingly transparent to the higher energy the $\mathrm{K}$ line from $\mathrm{Au}$ at had low intensities. Additional $\mathrm{Pb}$ shielding was added to prevent the $\mathrm{Lu}$ fluorescence from reaching the HEXITEC detector. This also meant that there were no X-rays above the $\mathrm{K}$-edge of $\mathrm{Cd}$ or Te so their fluorescence lines were also removed from the spectra. Figure 2 (b) shows the number of counts per pixel when the 
spectra per pixel were integrated from $9 \mathrm{keV}$ to $13 \mathrm{keV}$ to cover the $\mathrm{L}$ lines from $\mathrm{Au}$. The image demonstrates that the configuration was able to resolve $125 \mu \mathrm{m}$ spaced features despite blurring from the $50 \mu \mathrm{m}$ pinhole.

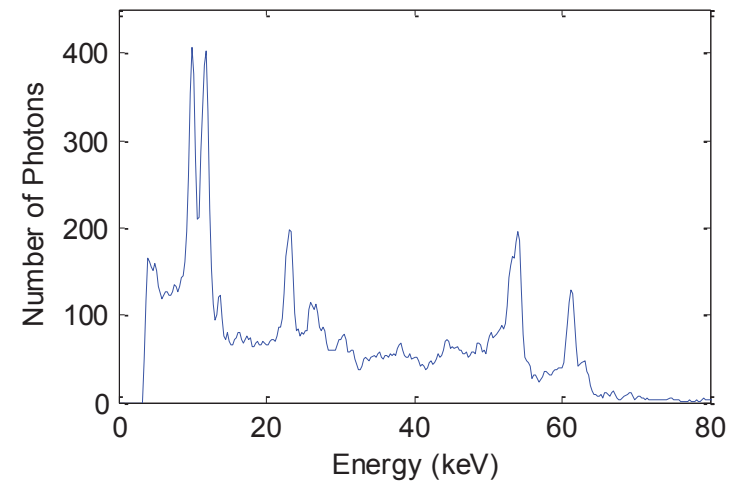

(a)

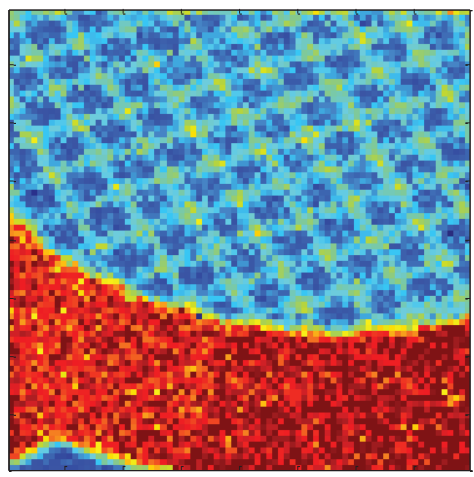

(b)

FIGURE 2. (a) A spectrum from $4 x 4$ pixels on the mesh frame summed together with the Au L lines in. (b) An image of the intensity per pixel from $9 \mathrm{keV}$ to $13 \mathrm{keV}$ to cover the $\mathrm{Au} \mathrm{L} \mathrm{line.}$

The sample was heated to $560^{\circ} \mathrm{C}$ and an image was collected in a region of the sample where there were $\mathrm{Zr}$, Mo and $\mathrm{Ag}$ rich regions. Figure 3 (a) shows a section of the transmission radiograph collected with an integration time of 0.008 s. Given the low flux of fluorescence X-rays the fluorescence data was collected for 60 s. Figure 3 (b) shows the spectrum from $3 \times 3$ pixels summed together from regions of the image that were rich in $\mathrm{Ag}$ (blue), Mo (red) and $\mathrm{Zr}$ (black). The $\mathrm{Cu}$ from the Al-Cu matrix was not visible as the $8.0 \mathrm{keV} \mathrm{Cu} \mathrm{K}_{\alpha}$ lines were heavily self-absorbed. The spectra per pixel were integrated over different energy ranges to provide images of the different elements. Images (c) to (e) had a median filter over a $3 \times 3$ pixel range applied to smooth the images. The $\mathrm{Zr}$ image in (c) was from the integration from $15 \mathrm{keV}$ to $16 \mathrm{keV}$ to cover the $\mathrm{Zr} \mathrm{K}_{\alpha}$ line only. The Mo image in (d) was from the integration from $17 \mathrm{keV}$ to $20 \mathrm{keV}$ to cover the $\mathrm{K}_{\alpha}$ and $\mathrm{K}_{\beta}$ lines. As the $\mathrm{K}_{\beta}$ line from $\mathrm{Zr}$ is at $17.7 \mathrm{keV}$ and the $\mathrm{Mo}_{\alpha}$ line is at $17.5 \mathrm{keV}$, they could not be separated and had to be corrected. The number of $\mathrm{Zr} \mathrm{K}$ events was used to estimate the expected number of $\mathrm{Zr} \mathrm{K}_{\beta}$ events [5], which were subtracted from the Mo image. This correction worked reasonably well but left some artefacts, for example in the bottom left hand corner of image (d). The Ag image in (e) was from the integration from $21 \mathrm{keV}$ to $25 \mathrm{keV}$ to cover the $\mathrm{Ag} \mathrm{K} \mathrm{K}_{\alpha}$ and $\mathrm{K}_{\beta}$ lines. This image and the spectra per pixel show that there were appreciable concentrations of $\mathrm{Ag}$ across the image due to the comparatively fast diffusion of $\mathrm{Ag}$ at this temperature, whereas the $\mathrm{Zr}$ and Mo were much slower to dissolve and diffuse at this temperature. Further details on the solidification experiments can be found in Liotti et al. [6].

\section{DISCUSSION AND SUMMARY}

This work has shown that the fluorescence imaging configuration with the energy discriminating imaging HEXITEC detector can image the elemental distribution of a sample with elements emitting fluorescence energies greater than $10 \mathrm{keV}$. The spectrum per pixel provided suitable images in $60 \mathrm{~s}$ exposures but this limitation was from the fluorescence flux which was $<200$ photons per pixel per minute. The detector would be capable of collecting up to 1000 photons/pixel/s that would allow images to be collected in $<1 \mathrm{~s}$. High imaging rates could also be achieved by summing the spectra of neighboring pixels at the expense of spatial resolution.

The spatial resolution was limited by the pinhole projection onto the HEXITEC detector. This means that feature sizes of $10-20 \mu \mathrm{m}$ appear to be the resolution limit to the technique. Although this would be too large for some applications there are many areas, for example in material science, where this feature size or larger would be applicable. Additionally the combination of high resolution transmission imaging and lower resolution fluorescence imaging could be combined to provide new insight even if the fluorescence information is at a lower resolution.

The field of view of the HEXITEC detector at the highest magnification was only $1 \mathrm{x} 1 \mathrm{~mm}^{2}$. HEXITEC detectors are butt-able on 3 sides and have been tiled in $2 \times 2$ [7] and $5 \times 5$ [8] detector modules with dead spaces of $<2$ pixels between the individual detectors. This method of creating a large focal plane detector could be used to create a larger field of view for fluorescence imaging instruments in the future. Unlike the scanning techniques, adding more pixels to the detector would not slow the imaging rate whilst increasing the FoV. 
Further optimization of experimental geometry and testing on the I12 Joint, Engineering, Environmental and Processing beamline at the Diamond Light Source are planned to demonstrate the potential at higher fluorescence energies and with a higher flux provided by the beamline. The suitability of imaging the elemental distribution on the 1-10s timescale with a spatial resolution on the order of $10 \mu \mathrm{m}$ or larger will be investigated with potential applications in metal alloy growth, geology, catalytic and chemical processing.

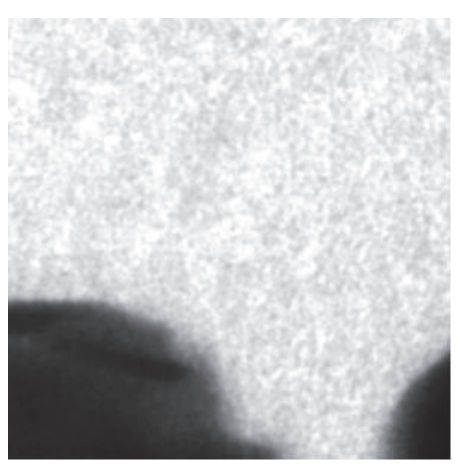

(a)

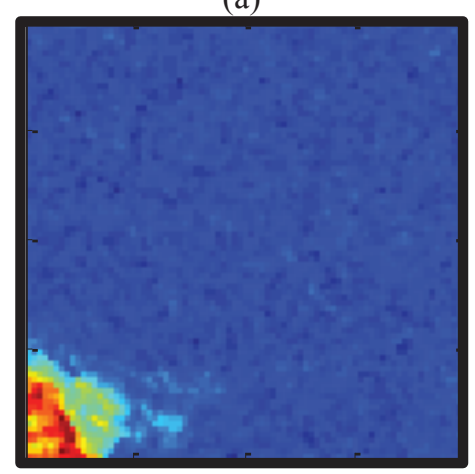

(c)

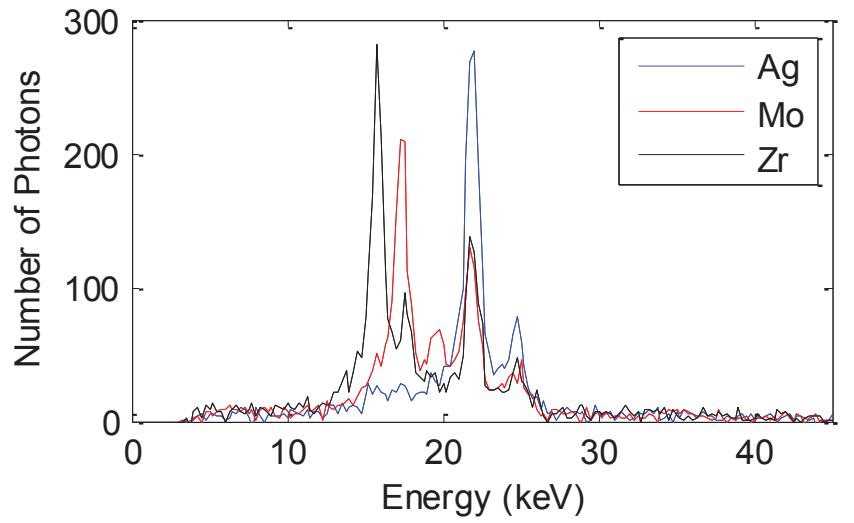

(b)

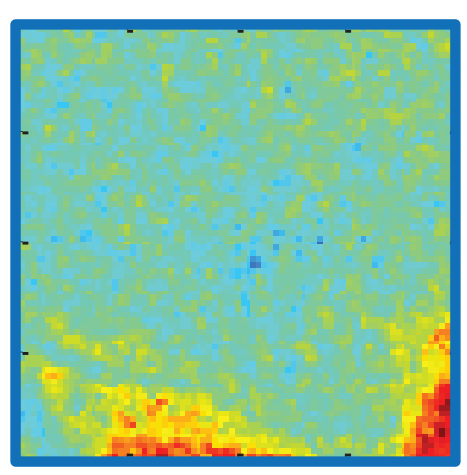

(e)

FIGURE 3. (a) The transmission radiograph image cropped and rotated to provide the same FoV as the fluorescence images. (b) The spectrum from $3 \times 3$ pixels summed together from areas that were Ag, Mo and $\mathrm{Zr}$ rich. (c) An image of the number of counts per pixel from the $\mathrm{Zr} \mathrm{K}_{\alpha}$ line only. (d) An image of the number of counts per pixel from the Mo $\mathrm{K}_{\alpha}$ and $\mathrm{K}_{\beta}$ lines. (e) An image of the number of counts per pixel from the $\mathrm{Ag} \mathrm{K}_{\alpha}$ and $\mathrm{K}_{\beta}$ lines.

\section{ACKNOWLEDGEMENTS}

PG, AL and EL would like to thank the UK Engineering and Physical Sciences Research Council for financial support through grant EP/H026177/1.

\section{REFERENCES}

1. Mirihanage, W.U. et al., Acta Materialia, 81, pp.241-247. 2014.

2. V. M. Egerton, et al., J. Anal. At. Spectrom., 30, 627-634, 2015.

3. P. Seller, et al., JINST, 6, C12009, 2011, doi: 10.1088/1748-0221/6/12/C12009

4. M. Veale, et al., Nucl. Inst. Meth. A, 767, 218-226, 2014, doi:10.1016/j.nima.2014.08.036

5. A. Thompson, et al., "X-ray Data Booklet", LBNL/PUB-490 Rev. 3, 2009.

6. E. Liotti, et. al, submitted to Scientific Reports, June 2015

7. M. D. Wilson, et al., IEEE Trans. Nucl. Sci, 60, 2, 2013.

8. M. D. Wilson, et al., Submitted to J. Inst, June 2015. 